
МИКРОНОДУЛЯРНОЙ ГИПЕРПЛАЗИИ НАДПОЧЕЧНИКОВ

\author{
( А. Шевэ*, Д.Г. Бельцевич, А.Ю. Абросимов, Д.А. Деркач, А.А. Лазарева
}

Национальный медицинский исследовательский центр эндокринологии, Москва, Россия

Микронодулярная гиперплазия надпочечников (МикГН) является редкой причиной АКТГ-независимого гиперкортицизма, которая может быть разделена по крайней мере на две отдельные группы патологий: первичная пигментированная МикГН и непигментированная МикГН, среди которых выделяют семейные и спорадические формы. Наиболее часто встречающейся является генетически детерминированная семейная форма первично-пигментированной МикГН как одна из составляющих комплекса Карни, обусловленная герминальной мутацией в гене $P R K A R 1 A$. При изолированных формах, помимо мутации в гене PRKAR1A, описаны инактивирующие мутации в генах, кодирующих фосфодиэстеразы (PDE11A4 и PDE8B), а также амплификация гена PRKACA.

Несмотря на относительную давность описания микронодулярной гиперплазии и одноименного комплекса Эйданом Карни в 1985 г., детальное изучение патофизиологических механизмов, генетических и клинических аспектов данной патологии, на сегодняшний день клиницисты продолжают сталкиваться с «нетипичными» случаями заболевания. Таким образом, природа данного заболевания недостаточно изучена и требует дальнейшего исследования. В данной обзорной статье представлены накопленные данные о МикГН с акцентом на генетические аспекты, а также описаны 2 уникальных клинических случая изолированной МикГН с результатами односторонней адреналэктомии.

КЛЮЧЕВЫЕ СЛОВА: синдром Иценко-Кушинга; микронодулярная гиперплазия надпочечников; односторонняя адреналэктомия.

\title{
CLINICAL AND GENETIC HETEROGENEITY OF MICRONODULAR ADRENAL HYPERPLASIA
}

\author{
() Anastassia Chevais*, Dmitriy G. Beltsevich, Aleksandr Yu. Abrosimov, Dmitriy A. Derkatch, Anna A. Lazareva
}

Endocrinology Research Centre, Moscow, Russia

Micronodular adrenal hyperplasia is a rare cause of ACTH-independent Cushing syndrome. It can be divided into two entities: primary pigmented nodular adrenocortical disease (PPNAD) and non-pigmented micronodular adrenocortical disease, among which familial and sporadic forms are distinguished. The most common is the genetically determined familial form PPNAD, as one of the components of Carney complex. The vast majority of patients have identifiable pathogenic variants in the PRKAR1A gene. In addition to the PRKAR1A gene mutations, inactivating mutations in the genes encoding phosphodiesterases (PDE11A4 and $P D E 8 B$ ), as well as PRKACA gene amplification, have been described in individuals with isolated forms. Despite the relative antiquity of the description of micronodular adrenal hyperplasia and the Carney comlex, a detailed study of pathophysiological mechanisms, genetic and clinical aspects of this pathology, nowadays, clinicians continue to face «atypical» cases. Thus, the nature of this disease is not well understood and requires further research. This review presents the accumulated data on micronodular adrenal hyperplasia, genetics aspects, and also describes 2 unique clinical cases of isolated PPNAD with unilateral adrenalectomy results.

KEYWORDS: primary pigmented nodular adrenocortical disease; micronodular adrenal hyperplasia; Cushing's syndrome; unilateral adrenalectomy.

\section{ВВЕДЕНИЕ}

Микронодулярная гиперплазия надпочечников (МикГН) - двустороннее поражение надпочечников, приводящее к избыточной продукции кортизола. МикГН является редкой патологией и составляет 0,6-1,9\% АКТГ-независимого гиперкортицизма (АКТГ - адренокортикотропный гормон), из которых около 70\% - семейные детерминированные формы [1, 2]. По данным ретроспективных исследований, заболеваемость превалирует у лиц молодого и среднего возраста (24,2士11,0 года), патология встречается чаще у женщин, чем у мужчин, соотношение 2,3:1 [3].

На основании гистологических данных Tirosh и соавт. предложили подразделить МикГН на три группы:

- первично-пигментированную МикГН в рамках синдрома Карни;
- изолированную первично-пигментированную МикГН, не связанную с комплексом Карни (и-МикГН);

- изолированную непигментированную МикГН.

Все три группы имеют спорадические и семейные формы [4].

Данная классификация двусторонних микронодулярных поражений надпочечников основывается на двух основных гистологических характеристиках: состояние коры - ее гиперплазия или атрофия и присутствие пигмента внутри узелков [5]. Макроскопически обнаруживают надпочечники нормального или несколько увеличенного размера, с множественными мелкими узелками различных оттенков от светлого до темно-коричневого цвета, диаметром 1-4 мм [4, 6, 7]. Окрашивание узелков происходит в результате накопления в них липофусцина - желто-коричневого пигмента, образующегося 
и накапливающегося в результате окислительного повреждения молекул липидов или в случае повреждения мембран органелл (митохондрий и лизосом). Микроскопически данные узелки представлены крупными клетками с небольшими ядрами, содержащими в эозинофильной цитоплазме гранулы липофусцина, митотическая активность в узелках очень низкая [7-9].

В основе патогенеза любой глюкокортикоид-продуцирующей опухоли надпочечника лежит нарушение в регуляции активности цАМФ-зависимой протеинкиназы А (ПКА; цАМФ - циклический аденозинмонофосфат). Генетические мутации вызывают нарушение в регуляции активности цАМФ-ПКА сигнального пути, приводя к чрезмерной активации ПКА и/или снижению активности фосфодиэстераз (ФДЭ), результатом чего является автономная продукция кортизола. Так, инактивирующая герминальная мутация ПКА-регуляторного гена-супрессора опухолевого роста R1a (PRKAR1A) ведет к повышению активности ПКА, в свою очередь, инактивирующие мутации в генах PDE11A4 и $P D E 8 B$, кодирующих ФДЭ, нарушают процесс гидролиза цАМФ и препятствуют инактивации ПКА. Сверхэкспрессия PRKACA, по-видимому, подавляет ингибирующую способность регуляторных единиц (R), тем самым вызывая цАМФ-независимую активацию ПКА $[4,10]$.

Клинические признаки синдрома Карни, ассоциированного с МикГН, характеризуются наличием кожных проявлений (лентигиноз, кожные и слизистые миксомы, голубые эпителиоидные невусы, пятна «кофе с молоком»), миксом сердца и молочных желез, опухолей яичек (крупноклеточные кальцинированные опухоли клеток (ертоли), псаммозных меланотических шванном, реже - соматотропином [1].

Диагностирование данной патологии принципиально не отличается от других состояний, сопровождающихся синдромом Кушинга (СК), и состоит из трех этапов. Первоочередно подтверждается наличие эндогенного гиперкортицизма (проведение ночного подавляющего теста с 1 мг дексаметазона (ПДТ1) и исключение экзогенного введения/поступления глюкокортикоидов (ГКС)) [11]. Некоторые авторы в качестве патогномоничного признака МикГН описывают парадоксальное повышение цифр кортизола на подавляющих тестах [2]. Вторым этапом следует определение АКТГ, уровень которого при данной форме СК снижен (<5 пг/мл). Заключительный этап включает визуализирующие методы исследования (мультиспиральную компьютерную томографию (МСКТ) и магнитно-резонансную томографию (МРТ)), основной целью которых является исключение макроопухолей как в надпочечниках, так и эктопированных образований в паранефральной клетчатке. Обнаружить микрогиперпластические изменения в коре надпочечников удается редко в связи с размерами надпочечников, которые в большинстве случаев находятся в пределах нормы или незначительно увеличены в основном за счет небольших узелков до 5 мм, изменяющих контуры надпочечников.

Молекулярно-генетическое обследование направлено на поиск мутаций генов PRKAR1A, PDE11A, PDE8B, CNC 2 locus, PRKACA. Анализ генетического сцепления показал, что существует как минимум два независимых локуса, ассоциированных с комплексом Карни: 17q22-24 (PRKAR1A) и 2p16 (CNC). Генетическое обследо- вание на данный момент предлагается в качестве скрининга в семьях с этими мутациями [12].

Основным методом лечения на сегодняшний день остается оперативное вмешательство. По данным американского эндокринологического сообщества (ААСЕ), оптимальным объемом оперативного лечения является лапароскопическая двусторонняя адреналэктомия [13]. Однако в ряде работ сообщается об эффективности односторонней адреналэктомии с сохранением стойкой ремиссии в отдаленном послеоперационном периоде $[14,15]$. Эффективность лечения оценивают как лабораторно (определение свободного кортизола в суточной моче, повторное проведение ПДт1), так и клинически, по степени регрессии симптомов СК (нормализации массы тела, артериального давления (АД), углеводного обмена, репродуктивной функции и т.д.) [6].

В клинической практике с МикГН чаще встречаются детские эндокринологи, так как в подавляющем большинстве случаев данная патология встречается у молодых людей в составе комплекса Карни $[4,16]$. В данной статье представлены два уникальных клинических случая с изолированной МикГН, диагностированной у пациентов в достаточно зрелом возрасте.

\section{КЛИНИЧЕСКИЙ СЛУЧАЙ №1}

Пациент М., 56 лет, страдающий тяжелым остеопорозом в течение последних 5 лет и имеющий множественные компрессионные переломы тел позвонков (Th6, Th9, Th12, L2, L4), обратился в ФГБУ «НМИЦ эндокринологии» с жалобами на выраженную слабость, быструю утомляемость, боли в поясничном отделе позвоночника, повышение АД по утрам до максимальных цифр 170/110 мм рт.ст.

Из анамнеза известно, что изменения внешности (увеличение окружности живота) и повышение АД максимально до 170/100 мм рт.ст. пациент начал отмечать около 5 лет назад. Диагноз АКТГ-независимого гиперкортицизма был поставлен в 2016 г. на основании лабораторно-инструментальных данных: кортизол в крови утром - 627 нмоль/л, в ходе GLN1 - 510 нмоль/л, кортизол в слюне вечером - 17,3 нмоль/л, базальный АКТГ - 1,9 пг/мл. С целью топической диагностики неоднократно проводилась МСКТ забрюшинного пространства с контрастным усилением, по данным которой патологических изменений надпочечников не выявлено. Специфического лечения не получал.

Из сопутствующей патологии: в 2017 г. диагностирован рак желудка (перстневидно-клеточный) T2N0M0 IB стадии, по поводу которого проведена дистальная субтотальная резекция желудка.

При физикальном обследовании отмечалось изменение внешности по кушингоидному типу - перераспределение подкожножировой клетчатки (ПЖК), «лунообразное» лицо, матронизм, мраморность кожи. Несмотря на нормальные показатели индекса массы тела (ИМТ) 24,2 кг/м² (вес 62 кг при росте 160 см) у пациента отмечалось выраженное центральное ожирение, сочетающееся с атрофией мышц конечностей и сглаженностью ягодичных мышц.

Также в ходе госпитализации проведено гормональное исследование крови и мочи, результаты приведены в табл. 1. 
Таблица 1. Данные лабораторных исследований пациента №1

\begin{tabular}{lccc}
\hline \multicolumn{1}{c}{ Показатель } & Результат & Единицы измерения & Референс \\
\hline Кортизол (кровь), утро & 632,2 & нмоль/л & $123-626$ \\
Кортизол (кровь), вечер & 644,5 & нмоль/л & $46-270$ \\
Кортизол (слюна), вечер & 22,93 & нмоль/л & $0,5-9,4$ \\
Кортизол св. (моча), сут & 1206,25 & нмоль/сут & $60-413$ \\
АКТГ (утро) & 1,13 & пг/мл & $7-66$ \\
ПДТ1 & 617,8 & нмоль/л & Менее 50 \\
\hline
\end{tabular}

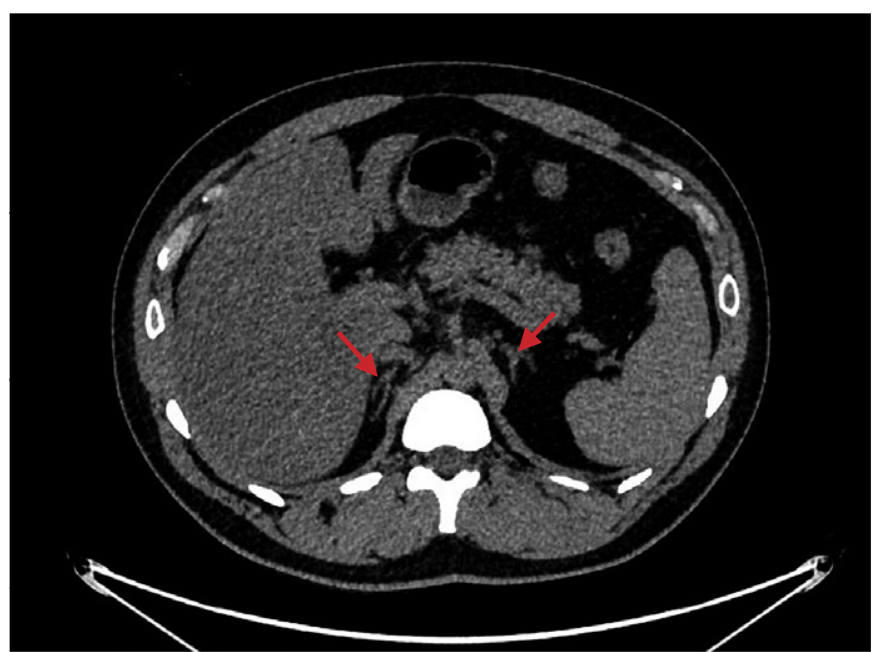

Рисунок 1. Мультиспиральная компьютерная томография надпочечников без контрастирования пациента №1.
В апреле 2019 г. пациенту выполнена эндоскопическая ретроперитонеальная правосторонняя адреналэктомия. В послеоперационном периоде клинических признаков надпочечниковой недостаточности $(\mathrm{HH})$ не наблюдалось. По данным гормонального анализа крови, кортизол (утро) - 524,4 нмоль/л, кортизол (вечер) - 599,9 нмоль/л, кортизол суточной порции мочи - 860,2 нмоль/сут, что свидетельствует о сохраняющемся АКТГ-независимом гиперкортицизме. Ввиду неэффективности односторонней операции запланирована левосторонняя адреналэктомия, которая откладывается в связи с некомплаентностью пациента.

По данным гистологического исследования послеоперационного материала: в доставленном материале наблюдаются фрагменты жировой клетчатки и надпочечника общими размерами 7,0×5,5×2,0 см (рис. 2). В коре визуализируются множественные коричневатые узелки до 0,4 см. При микроскопии тканевая архитектоника коры
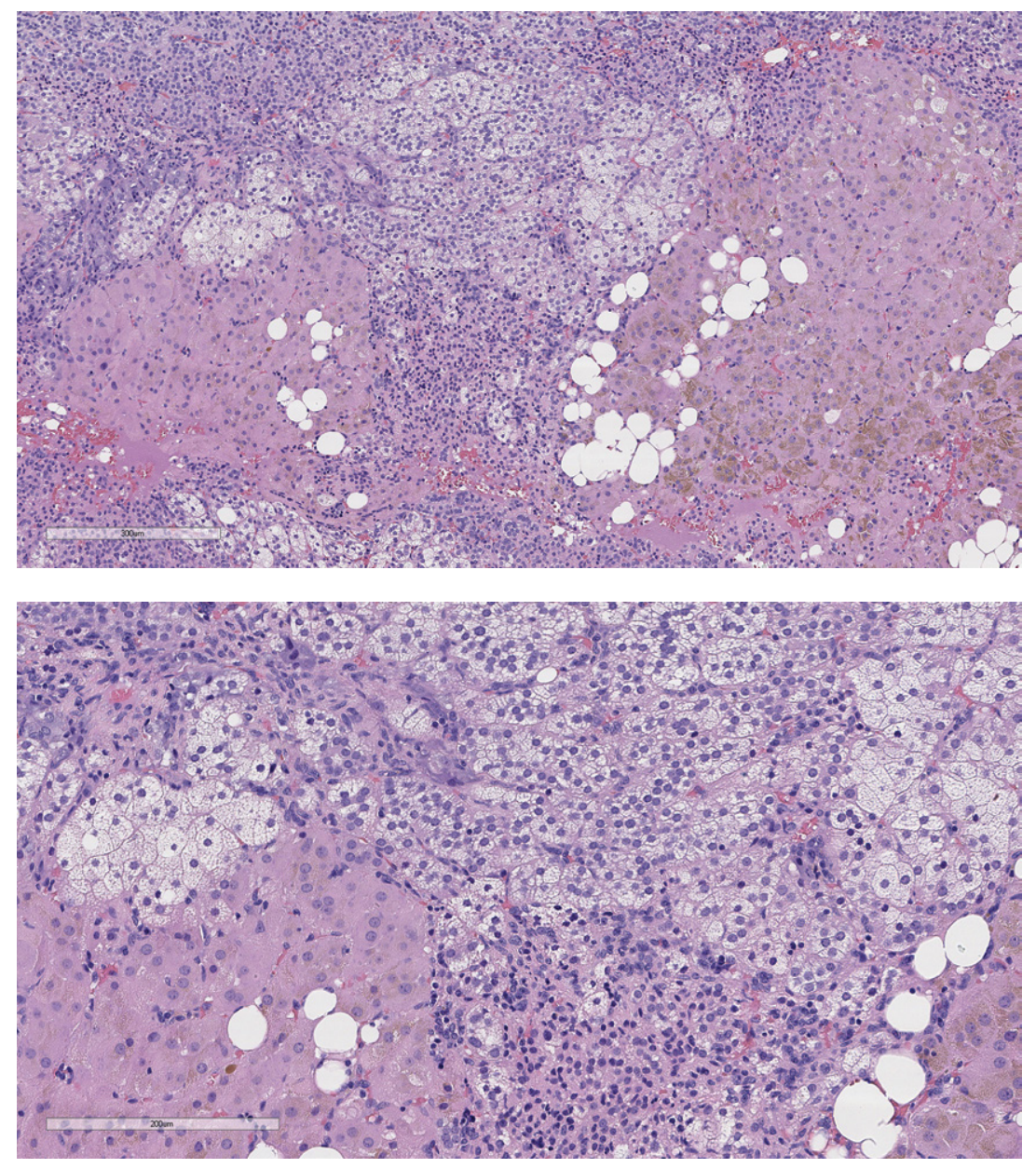

Рисунок 2. Гистологическая картина послеоперационного материала надпочечника пациента №1. 
надпочечника нарушена за счет образования множества мелких узелков из разных клеток. Большая часть клеток с компактной эозинофильной цитоплазмой, содержащей коричневатый пигмент (липофусцин) и часть светлых клеток, богатых липидами. Обнаруженная патоморфологическая картина может соответствовать первичной пигментно-узелковой адренокортикальной болезни.

\section{КЛИНИЧЕСКИЙ СЛУЧАЙ №2}

Пациентка С., 40 лет, в течение многих лет наблюдалась у кардиолога в связи с нецелевыми и нестабильными цифрами АД (повышение до 180/110 мм рт. ст.), по поводу чего получала многокомпонентную гипотензивную терапию (ингибиторами ангиотензинпревращающего фермента (иАПФ), бета-адреноблокаторами (ББ), петлевыми диуретиками) с положительным эффектом. За последние 5 лет отмечает изменение внешности (увеличение массы тела, перераспределение ПЖК с преобладанием в области лица и живота, избыточный рост волос на верхней губе, на животе), нарушение менструального цикла. Из анамнеза известно, что пациентка перенесла два низкотравматичных перелома VII и IX ребер, остеоденситометрия не проводилась. Учитывая стойкое повышение АД, нарушение менструального цикла, нарушенную толерантность к глюкозе и данные физикального обследования, первоначально был заподозрен и впоследствии подтвержден диагноз эндогенного гиперкортицизма, АКТГ-независимая форма.

Данные осмотра при поступлении: ИМТ - 27,6 кг/м² (вес 69 кг при росте 158 см). Перераспределение ПЖК по кушингоидному типу с наибольшим преобладанием в области живота, скошенные ягодицы. На боковых поверхностях живота определяются стрии бледно-розового цвета. Лунообразное лицо.

В ходе госпитализации проведено лабораторное исследование, результаты приведены в таблице 2.

Выполнена МСКТ органов забрюшинного пространства, по данным которой определяется узелковая гиперплазия левого надпочечника, нельзя исключить узелковую гиперплазию правого надпочечника (рис. 3).

Учитывая наличие у пациентки низкотравматичных переломов в анамнезе, в ходе госпитализации проведена остеоденситометрия, по данным которой определяется снижение МПК в поясничном отделе позвоночника (до -1,8 SD по Z-критерию в L1-L4), лучевой кости (до -1,3 SD по Z-критерию в ультрадистальном отделе) и в проксимальном отделе бедренной кости (до -0,5 SD по Z-критерию Total), что соответствует остеопении.

В апреле 2019 г. пациентке выполнена эндоскопическая левосторонняя адреналэктомия. В связи с развитием НН в раннем послеоперационном периоде инициирована гормональная заместительная терапия с последующей коррекцией терапии (гидрокортизон 15 мг/сут).

По данным гистологического исследования послеоперационного материала подтверждена МикГН. Макроскопически надпочечник содержит множество фрагментов жировой ткани от 1,5 до 3,0 см в наибольшем измерении с участками желтовато-коричневого цвета, представляющими ткань надпочечника. Микроскопическое описание: Фрагменты ткани надпочечника с множественными очагами гиперплазии клеток коркового слоя с широкой оксифильной цитоплазмой (рис. 4).

Таблица 2. Гормональный анализ крови и мочи пациентки №2

\begin{tabular}{lccc}
\hline \multicolumn{1}{c}{ Показатель } & Результат & Единицы измерения & Референс \\
\hline Кортизол (слюна), вечер & 9,8 & нмоль/л & $0,5-9,4$ \\
Кортизол св. (моча), сут & 215,2 & нмоль/сут & $60-413$ \\
АКТГ (утро) & 1 & пг/мл & $7-66$ \\
ПДТ1 & 470,8 & нмоль/л & Менее 50 \\
\hline
\end{tabular}
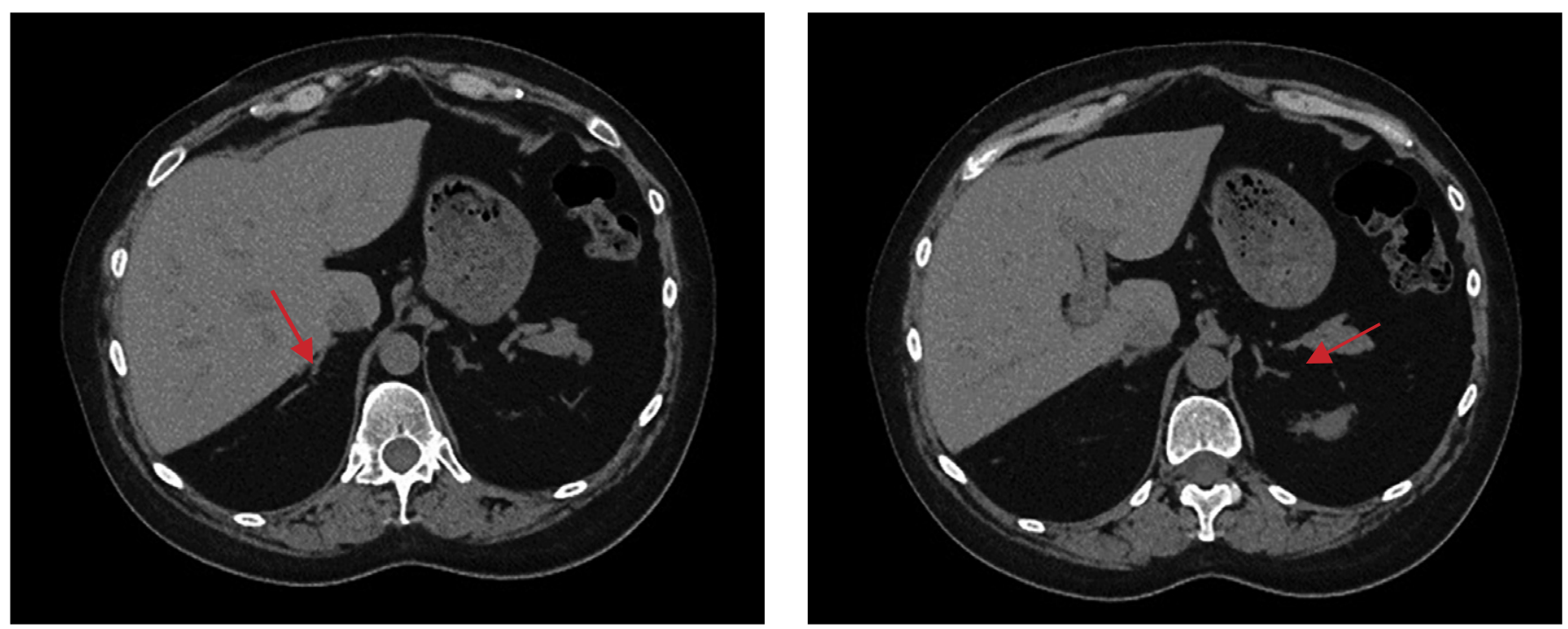

Рисунок 3. Мультиспиральная компьютерная томография надпочечников без контрастирования пациентки №2. 

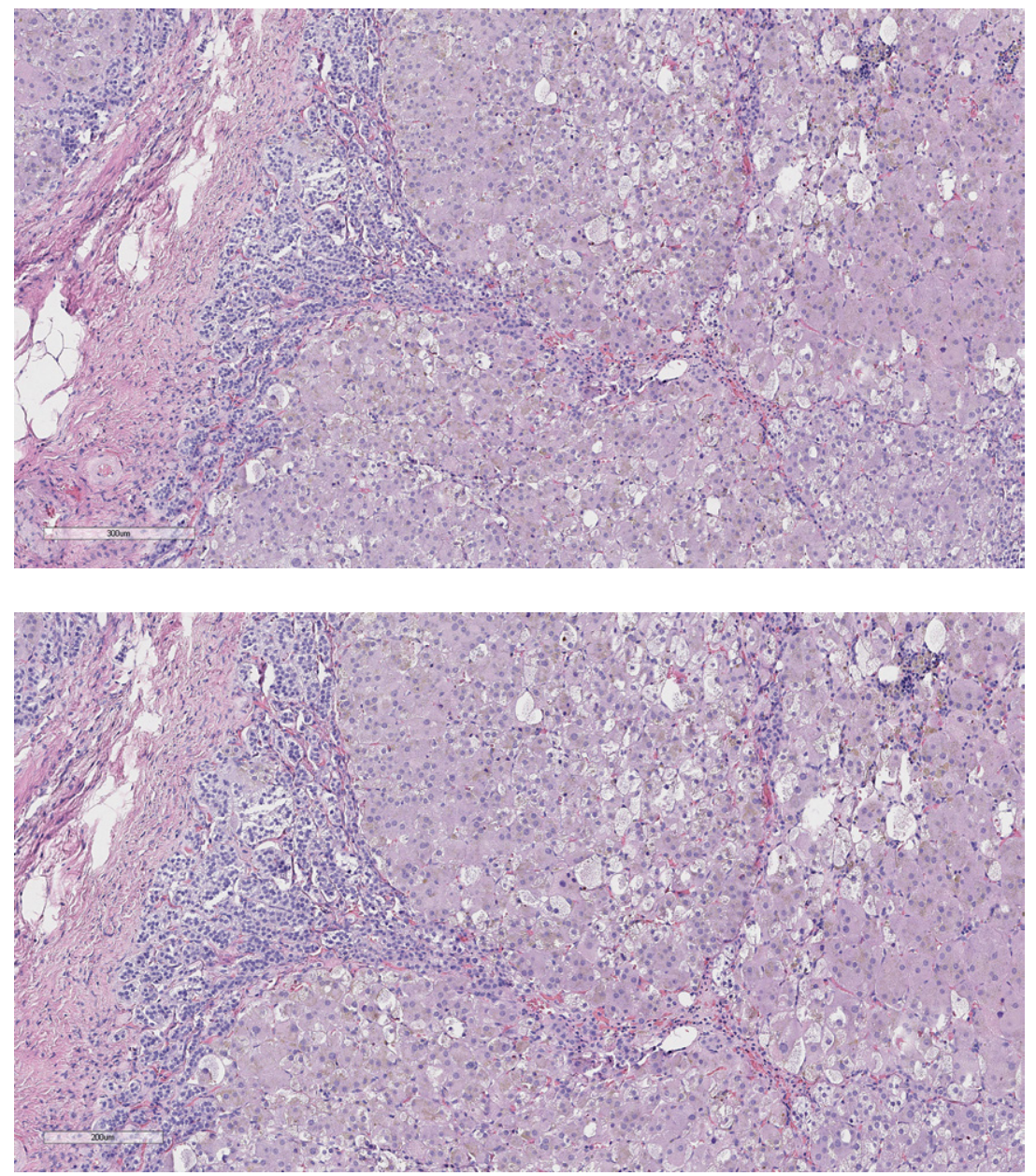

Рисунок 4. Гистологическая картина послеоперационного материала надпочечника пациентки №2.

\section{ОБСУЖДЕНИЕ}

Двусторонняя МикГН является орфанным заболеванием. Редкость подобных заболеваний вызывает трудности их своевременного диагностирования и лечения. Данный диагноз является, скорее, диагнозом исключения, так как отсутствуют привычные для АКТГ-независимого гиперкортицизма опухолевые изменения в надпочечниках на МСКТ и есть абсолютно несомненные лабораторные данные, свидетельствующие об АКТГ-независимом гиперкортицизме. Приведенные нами клинические случаи еще раз доказывают сложность своевременной диагностики и принятия однозначного решения о выборе объема хирургического вмешательства.

Во-первых, чаще всего данная патология встречается в составе синдрома множественных эндокринных неоплазий 4 типа (синдрома Карни), однако в нашем случае оба пациента имеют изолированную пигментированную надпочечниковую гиперплазию. При тщательном обследовании других компонентов комплекса Карни (лентигиноз, миксомы, голубые эпителиоидные невусы, пятна «кофе с молоком» и др.) выявлено не было.

Заболевание обычно поражает лиц более молодого

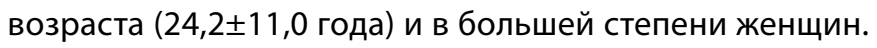
Учитывая этот факт, второй диагностической сложностью стал возраст обоих пациентов, в особенности пациента №1, у которого он составил 56 лет. Можно предположить, что изолированная форма первичнопигментированной МикГН ассоциирована с наиболее поздней манифестацией заболевания, нежели в составе комплекса Карни.

Во многих работах при проведении гормональных исследований отмечались парадоксальные повышения цифр кортизола в ходе ПДТ1, однако в приведенных нами клинических наблюдениях ожидаемого патогномоничного признака выявлено не было.

Следующим и наиболее важным пунктом, безусловно, является объем оперативного вмешательства. Как было упомянуто ранее, рекомендованный объем операции, по данным Американской ассоциации эндокринологов, - двусторонняя адреналэктомия. Однако, принимая во внимание описанные случаи успешной односторонней адреналэктомии с последующей многолетней ремиссией, было принято решение об односторонней адреналэктомии у обоих пациентов. Мы получили два противоположных результата: в первом случае ремиссии заболевания достигнуто не было, в то время как у пациентки №2 в раннем послеоперационном периоде развилась стойкая НН, что являлось более благоприятным признаком и свидетельствовало об успешном исходе оперативного лечения. Несомненно, период наблюдения данной пациентки был непродолжительным, чтобы сделать однозначный вывод об эффективности односторонней адреналэктомии, в связи с чем требуется дальнейшее наблюдение для определения динамики состояния и длительности ремиссии. Возможным объяснением неэффективности данного объема хирургического вмешательства у пациента №1 являются первоначально 
более выраженные проявления СК на дооперационном этапе. Также необходимо учитывать данные MCКТ-исследований: у пациента №1 оба надпочечника были в пределах нормы и значительно не отличались по размеру, в то время как у пациентки №2 поражение было более выражено с одной стороны.

Учитывая снижение качества жизни пациента и высокие риски смерти от острой НН после проведения билатеральной адреналэктомии, мы считаем целесообразным выполнение односторонней адреналэктомии с последующей клинической и лабораторной оценкой стероидного обмена. В случае неэффективности первой операции показано проведение тотальной адреналэктомии.

Перспективным и, возможно, прогностически ценным будет проведение молекулярно-генетического исследования генов (PRKAR1A, PDE11A4, PDE8B, PRKACA) у пациентов с клиническим диагнозом МикГН.

\section{МОЛЕКУЛЯРНО-ГЕНЕТИЧЕСКАЯ ДИАГНОСТИКА: ДОСТИЖЕНИЯ И ПЕРСПЕКТИВЫ}

Как было упомянуто выше, в основе МикГН лежат генетические изменения, нарушающие регуляцию активности цАМФ-ПКА сигнального пути, которые приводят либо к чрезмерной активации ПКА, либо снижению активности ее дезактиватора ФДЭ.

Известны два изомера ПКА (1 и 2 типы), а также 4 изоформы регуляторной субъединицы (R1a, R1 $\beta, R 2 a, R 2 \beta)$ и 3 изоформы каталитической субъединицы (Ca, C $\beta, C \gamma)$. Каждую изоформу кодирует собственный ген [1]. Соответственно, три гена кодируют каталитические субъединицы ПКА (PRKACA, -CB и -CG) и четыре гена кодируют регуляторные субъединицы (PRKAR1A, -1B, -2A и -2B) [17]. Так, герминальная мутация ПКА-регуляторного гена-супрессора опухолевого роста R1a (PRKAR1A) ведет к повышению активности ПКА.

К инактивирующим относятся мутации в генах, кодирующих фосфодиэстеразы (PDE11A4 и PDE8B). При данных мутациях нарушается процесс гидролиза цАМФ, что препятствует воссоединению регуляторных и каталитических субъединиц ПКА. Амплификация гена PRKACA приводит к снижению ингибирующей способности регуляторных единиц ПКА, тем самым вызывая цАМФ-независимую активацию ПКА [4, 10].

Пораженные гены/локусы при Карни-комплексе:

\section{PRKAR1A и 2p16}

Анализ генетического сцепления показал, что существует как минимум 2 независимых локуса, ассоциированных с комплексом Карни: 17q22-24 и 2р16 [12, 18].

В2000г.был идентифицированген регуляторной субъединицы альфа (R1a) протеинкиназы A (PRKAR1A) [19]. Этот ген находится на длинном плече 17 хромосомы (17q22-24), и около 80\% больных с МикНГ в составе комплекса Карни имеют гетерозиготную мутацию данного гена [12]. Приблизительно 70\% из них - семейные формы, а оставшиеся случаи представляют собой мутацию de novo. Пенетрантность среди носителей мутаций PRKAR1A практически абсолютна и составляет 97,5\% [4].

PRKAR1A выступает в качестве классического генасупрессора опухолей. Онкогенез в опухолях при комплексе Карни обусловлен полным отсутствием функ- ционального гена PRKAR1A, приводящего к нарушению регуляции циклинов D-типа [19]. В работе Kirschner и coавт. было выявлено, что при потере нормального аллеля (в данном случае в 17q22-23) происходит утрата гетерогенности, что является распространенным явлением при злокачественных опухолях и указывает на отсутствие функционального гена-супрессора опухоли в потерянной области $[20,21]$. В данном случае нокаут данного аллеля приводит к повышению уровня циклинов D-типа.

Герминальные мутации PRKAR1A в подавляющем большинстве случаев определяются в 2, 3, 5, 7 и 8-м экзонах, на долю остальных приходятся интронные и сплайсинговые мутации [20]. Практически все мутации являются уникальными, только две: 491_492delTG в 5 экзоне и с.709-2_709-7delATTTTT в 7 интроне относят к мутационным «горячим точкам» (mutational hot spot) - локусам генома, характеризующимся большей мутабельностью [12]. Превалирующее число мутаций представлено заменой нуклеотида, делециями, вставками или комбинированными изменениями, включающими до 15 нуклеиновых кислот, однако в определенных работах, в частности Salpea и соавт. и Horvath и соавт., также описаны случаи и более крупных, хромосомных делеций у 11 и 2 неродственных пациентов с комплексом Карни соответственно [20, 22, 23].

Мутации были распределены по группам в соответствии с их молекулярными последствиями. К первой группе, составляющей более $90 \%$ всех мутаций, относят те изменения последовательности нуклеотидов, результатом которых является преждевременный стоп-кодон. Неправильно сплайсированные мРНК (содержащие стоп-кодоны в нетипичных местах) подвергаются нонсенс-опосредованному распаду (NMD) [24]. Данный процесс препятствует синтезу мутантного белка, что приводит к снижению уровня R1a более чем на $50 \%$ и, соответственно, повышению активности ПКА, что, в свою очередь, приводит к увеличению образованию ГКС [20].

Ко второй группе относят миссенс-мутации короткие вставки/делеции внутри рамки считывания, или сплайс-варианты, которые являются достаточно редкой причиной болезни. Миссенс-мутации находятся в конце гена и приводят к образованию дефектного белка, который не может должным образом взаимодействовать с цАМФ или неэффективно связывается с каталитическими субъединицами ПКА $[4,25,26]$.

Однако сохранялся вопрос о причинах выраженной вариабельности течения МикГН и реализации других опухолей у членов одной и той же семьи с PRKAR1A мутацией. Предположено, что большинство корреляций генотип-фенотип объяснимы наличием дополнительных генетических факторов, которые модулируют экспрессию данного признака, в связи с чем при изучении моногенных заболеваний внимание исследователей было обращено к генам-модификаторам. Libé и соавт. было выявлено, что PDE11A является геном, влияющим на фенотип у пациентов с мутацией PRKAR1A. В данной работе у $25 \%$ пациентов с герминативной мутацией PRKAR1A были обнаружены сочетанные мутации гена PDE11A. При этом дефекты PDE11A у пациентов с МикГН и сертоли-клеточными кальцифицированными опухолями наблюдались значительно чаще, чем у пациентов без этих проявлений. Носителями данной мутации чаще всего были мужчины [27]. 
Ген на 2-й хромосоме (2p16) до сих пор не установлен. Наличие альтераций (чаще всего амплификаций) в локусе 2p16-21 предполагает наличие в нем онкогена, отвечающего за канцерогенез у пациентов с комплексом Карни. Возможно, альтерации в данном локусе могут быть одним из самых ранних событий в патогенезе развития опухолевого роста при комплексе Карни [28].

Пораженные гены/локусы при изолированной форме МикГH: PRKAR1A, PRKACA, PDE11A

По сравнению с другими мутациями, описанными в гене PRKAR1A, герминальная мутация с. 709-7del6 в 6-м интроне практически всегда ассоциирована с изолированной формой первично-пигментированной МикГН. У всех пациентов с данной делецией наблюдалась и-МикГН, при обследовании других проявлений Карни-комплекса выявлено не было [29]. При и-МикГН большинство пациентов имеют данную мутацию, на долю остальных приходится миссенс-мутация Met1Val в гене PRKAR1A [30].

Амплификация гена PRKACA, кодирующего каталитическую субъединицу цАМФ-зависимой ПКА, ведет к утрате регуляции ее активности (происходит нарушение взаимодействия регуляторной-каталитической субъединиц при сохранении ферментативной активности ПКА). В исследовании наблюдались как дупликации, так и трипликации PRKACA, причем последние были ассоциированы с более ранней манифестацией заболевания и более тяжелым течением СК [31].

И, наконец, мутация гена $P D E 11 A$, расположенного на хромосоме 2q31.2 и кодирующего ФДЭ 11A, которая относится к большому семейству ФДЭ и обладает двойной специфичностью: расщепляет как цАМФ, так и циклический гуанозинмонофосфат [32, 33]. У пациентов с инактивирующими мутациями PDE11A была обнаружена потеря аллеля 2q, что приводило к значительному повышению уровня цАМФ и цГМФ в опухоли. Это позволило сделать вывод, что мутация в PDE11A предрасполагает к образованию МикГН и связана с неадекватной активацией цАМФ аденилаткиназного пути, так же как и уменьшение PDE11А мПКА и экспрессии белка $[32,34]$.
Пораженные гены/локусы при изолированной форме непигментированной МикГН: PDE8B, PDE11A, PRKACA

PDE8B является одним из регуляторов стероидогенеза надпочечников. Хотя механизм действия до конца не изучен, предполагается, что в норме в коре надпочечников фосфодиэстеразы 8В действует как ингибитор стероидогенеза [33]. Локус для цАМФ-специфической ФДЭ (PDE8B) находится на длинном плече 5 хромосомы (5q13) [35]. Однако, учитывая редкость данной патологии, на данный момент получено недостаточно данных о типе мутаций в гене $P D E 8 B$ и о характере их влияния на опухолевые образования надпочечников.

Описаны 3 случая амплификации PRKACA и 5 пациентов с мутацией в гене PDE11A. На данный момент мутаций в гене PRKAR1A при данной форме МикГН описано не было [4, 31, 34].

\section{ЗАКЛЮЧЕНИЕ}

МикГН - редкое и сложное заболевание, требующее не только особенного клинического подхода, но также и обязательного генетического анализа. Дальнейшие исследования позволят выявить новые генетические причины, регуляторные механизмы, участвующие в аномальном стероидогенезе при МикГН, на основании которых возможно будет формирование персонализированного подхода в выборе лечебной тактики.

\section{ДОПОЛНИТЕЛЬНАЯ ИНФОРМАЦИЯ}

Источник финансирования. Работа выполнена по инициативе авторов без привлечения финансирования.

Конфликт интересов. Авторы декларируют отсутствие явных и потенциальных конфликтов интересов, связанных с публикацией настоящей статьи.

Участие авторов. Все авторы одобрили финальную версию статьи перед публикацией, выразили согласие нести ответственность за все аспекты работы, подразумевающую надлежащее изучение и решение вопросов, связанных с точностью или добросовестностью любой части работы

Согласие пациента. Пациенты добровольно подписали информированное согласие на публикацию персональной медицинской информации в обезличенной форме в журнале «Эндокринная хирургия».

\section{СПИСОК ЛИТЕРАТУРЫ | REFERENCES}

1. Орлова Е.М., Карева М.А. Карни-комплекс - синдром множественных эндокринных неоплазий // Проблемы эндокринологии. - 2012. - Т. 58. - №3. - C. 22-30. [Orlova EM Kareva MA. Carney complex - multiple endocrine neoplasia syndrome. Problems of Endocrinology. 2012;58(3):22-30 (In Russ.)]. doi: https://doi.org/10.14341/probl201258322-30

2. Chen S, Li R, Lu L, et al. Efficacy of dexamethasone suppression test during the diagnosis of primary pigmented nodular adrenocortical disease in Chinese adrenocorticotropic hormoneindependent Cushing syndrome. Endocrine. 2018;59(1):183-190. doi: https://doi.org/10.1007/s12020-017-1436-9

3. Zhou J, Zhang M, Bai X, et al. Demographic Characteristics, Etiology, and Comorbidities of Patients with Cushing's Syndrome: A 10-Year Retrospective Study at a Large General Hospital in China. Int J Endocrinol. 2019:1-10. doi: https://doi.org/10.1155/2019/7159696

4. Tirosh A, Valdés N, Stratakis CA. Genetics of micronodular adrenal hyperplasia and Carney complex. Presse Med. 2018;47(7-8):127-137. doi: https://doi.org/10.1016/j.lpm.2018.07.005

5. Horvath A, Stratakis CA. Unraveling the molecular basis of micronodular adrenal hyperplasia. Curr
Opin Endocrinol Diabetes Obes. 2008;15(3):227-233. doi: https://doi.org/10.1097/MED.0b013e3282fe7416

6. Carney JA, Gordon H, Carpenter PC, et al. The complex of myxomas, spotty pigmentation, and endocrine overactivity. Medicine (Baltimore). 1985;64(4):270-283. doi: https://doi.org/10.1097/00005792-198507000-00007

7. Duan K, Hernandez KG, Mete O. Clinicopathological correlates of adrenal Cushing's syndrome.

Postgrad Med J. 2015:91(1076):331-342.

doi: https://doi.org/10.1136/postgradmedj-2014-202612rep

8. Ефимов А.А., Маслякова Г.Н. О роли липофусцина в инволютивных и патологических процессах. Саратовский научно-медииинский журнал. - 2009. - Т. 5. - №1. - C. 111-115. [Efimov AA, Maslyakova GN. Lipofuscin role in involutive and pathological processes. Saratov Journal of Medical Scientific Research. 2009;5(1):111-115 (In Russ.)].

9. Патологическая анатомия. Частная анатомия / Под ред. Паукова В.С. - М.: ГЭОТАР-Медиа; 2015. [Patologitcheskaya anatomiya. Tchastnaya anatomiya. Ed by Paukov VS. Moscow: GEOTAR-Media; 2015. (In Russ.)]. 
10. Mete O, Duan K. The Many Faces of Primary Aldosteronism and Cushing Syndrome: A Reflection of Adrenocortical Tumor Heterogeneity. Front Med (Lausanne). 2018:5:54. doi: https://doi.org/10.3389/fmed.2018.00054

11. Трошина Е.А., Бельцевич Д.Г., Молашенко Н.В., Газизова Д.О Диагностика, дифференциальная диагностика и лечение эндогенного гипер кортицизма // Проблемы Эндокринологии. 2010. - T. 56. - №2. - C. 53-63. [Troshina EA, Beltsevich DG, Molashenko NV, Gazizova DO. Diagnosis, differential diagnosis, and trea.ment of endogenous hypercorticism. Problems of endocrinology. 2010;56(2):53-63. (In Russ.)]. doi: https://doi.org/10.14341/probl201056253-63

12. Bertherat J, Horvath $A$, Groussin L, et al. Mutations in regulatory subunit type $1 \mathrm{~A}$ of cyclic adenosine $5^{\prime}$-monophosphate-dependent protein kinase (PRKAR1A): phenotype analysis in 353 patients and 80 different genotypes. J Clin Endocrinol Metab. 2009;94(6):2085-2091. doi: https://doi.org/10.1210/jc.2008-2333

13. Nieman LK, Biller BM, Findling JW, et al. Treatment of Cushing's Syndrome: An Endocrine Society Clinical Practice Guideline. J Clin Endocrino/ Metab. 2015;100(8):2807-2831. doi: https://doi.org/10.1210/jc.2015-1818

14. Xu Y, Rui W, Qi Y, et al. The role of unilateral adrenalectomy in corticotropin-independent bilateral adrenocortical hyperplasias. World J Surg. 2013;37(7):1626-1632. doi: https://doi.org/10.1007/s00268-013-2059-9

15. Memon SS, Thakkar K, Patil V, et al. Primary pigmented nodular adrenocortical disease (PPNAD): single centre experience. J Pediatr Endocrinol Metab. 2019;32(4):391-397. doi: https://doi.org/10.1515/jpem-2018-0413

16. Espiard S, Bertherat J.PPNAD, Carney complex and other micronodular adrenal hyperplasia. In: Huhtanieniemi I, Martini, editors. Reference Module in Biomedical Sciences. 2018. p. 271-282.

17. Vélayoudom-Céphise FL, Haissaguerre M, Tabarin A. Etiopathogeny of Primary Adrenal Hypercortisolism. Front Horm Res. 2016;46:39-53. doi: https://doi.org/10.1159/000443863

18. Stratakis CA, Carney JA, Lin JP, et al. Carney complex, a familial multiple neoplasia and lentiginosis syndrome: Analysis of 11 kindreds and linkage to the short arm of chromosome 2. J Clin Invest. 1996;97:699-705. doi: https://doi.org/10.1172/JCl118467

19. Nadella KS, Kirschner LS. Disruption of protein kinase a regulation causes immortalization and dysregulation of D-type cyclins. Cancer Res. 2005;65(22):10307-10315. doi: https://doi.org/10.1158/0008-5472.CAN-05-3183

20. Kirschner LS, Carney JA, Pack SD, et al. Mutations of the gene encoding the protein kinase A type I-a regulatory subunit in patients with the Carney complex. Nat Genet. 2000;26(1):89-92. doi: https://doi.org/10.1038/79238

21. Espiard S, Ragazzon B, Bertherat J. Protein Kinase A Alterations in Adrenocortical Tumors. Horm Metab Res. 2014;46(12):869-875. doi: https://doi.org/10.1055/s-0034-1385908

22. Horvath A, Bossis I, Giatzakis C, et al. Large Deletions of the PRKAR1A Gene in Carney Complex. Clin Cancer Res. 2008;14(2):388-395. doi: https://doi.org/10.1158/1078-0432.CCR-07-1155

23. Salpea P, Horvath A, London E, et al. Deletions of the PRKAR1A Locus at 17q24.2-q24.3 in Carney Complex: Genotype-
Phenotype Correlations and Implications for Genetic Testing. J Clin Endocrinol Metab. 2014;99(1):E183-E188 doi: https://doi.org/10.1210/jc.2013-3159

24. He F, Jacobson A. Nonsense-mediated mrna decay: degradation of defective transcripts is only part of the story. Annu Rev Genet. 2015;49(1):339-366. doi: https://doi.org/10.1146/annurev-genet-112414-054639.

25. Greene EL, Horvath AD, Nesterova M, et al. In vitro functional studies of naturally occurring pathogenic PRKAR1A mutations that are not subject to nonsense mRNA decay. Hum Mutat. 2008;29(5):633-639. doi: https://doi.org/10.1002/humu.20688

26. Meoli E, Bossis I, Cazabat L, et al. Protein kinase A effects of an expressed PRKAR1A mutation associated with aggressive tumors. Cancer Res. 2008;68(9):3133-3141. doi: https://doi.org/10.1158/0008-5472.CAN-08-0064

27. Libé R, Horvath A, Vezzosi D, et al. Frequent Phosphodiesterase $11 \mathrm{~A}$ Gene ( PDE1 1A ) Defects in Patients with Carney Complex (CNC) Caused by PRKAR1A Mutations: PDE1 1A May Contribute to Adrenal and Testicular Tumors in CNC as a Modifier of the Phenotype. J Clin Endocrinol Metab. 2011;96(1):E208-E214. doi: https://doi.org/10.1210/jc.2010-1704

28. Matyakhina L, Pack S, Kirschner LS, et al. Chromosome 2 (2p16) abnormalities in Carney complex tumours. J Med Genet. 2003:40(4):268-277. doi: https://doi.org/10.1136/jmg.40.4.268.

29. Groussin L, Horvath A, Jullian E, et al. A PRKAR1A Mutation Associated with Primary Pigmented Nodular Adrenocortical Disease in 12 Kindreds. J Clin Endocrinol Metab. 2006:91 (5):1943-1949. doi: https://doi.org/10.1210/jc.2005-2708

30. Pereira AM, Hes FJ, Horvath A, et al. Association of the M1VPRKAR1A Mutation with Primary Pigmented Nodular Adrenocortical Disease in Two Large Families. J Clin Endocrinol Metab. 2010:95(1):338-342. doi: https://doi.org/10.1210/jc.2009-0993

31. Lodish MB, Yuan B, Levy I, et al. Germline PRKACA amplification causes variable phenotypes that may depend on the extent of the genomic defect: molecular mechanisms and clinical presentations. Eur J Endocrinol. 2015:172(6):803-811. doi: https://doi.org/10.1530/eje-14-1154

32. Boikos SA, Horvath A, Heyerdahl S, et al. Phosphodiesterase $11 \mathrm{~A}$ expression in the adrenal cortex, primary pigmented nodular adrenocortical disease, and other corticotropinindependent lesions. Horm Metab Res. 2008;40(5):347-353. doi: https://doi.org/10.1055/s-2008-1076694

33. Vezzosi D, Bertherat J. Phosphodiesterases in endocrine physiology and disease. Eur J Endocrinol. 2011;165(2):177-188. doi: https://doi.org/10.1530/eje-10-1123

34. Horvath A, Giatzakis C, Robinson-White A, et al. Adrenal Hyperplasia and Adenomas Are Associated with Inhibition of Phosphodiesterase 11A in Carriers of PDE1 1A Sequence Variants That Are Frequent in the Population. Cancer Res. 2006;66(24):11571-11575. doi: https://doi.org/10.1158/0008-5472.can-06-2914

35. Horvath A, Mericq V, Stratakis CA. Mutation inP DE8B,a Cyclic AMP-Specific Phosphodiesterase in Adrenal Hyperplasia. New England Journal of Medicine. 2008;358(7):750-752 doi: https://doi.org/10.1056/nejmc0706182

\section{ИНФОРМАЦИЯ ОБ АВТОРАХ [AUTHORS INFO]}

*Шевэ Анастасия, аспирант [Anastassia Chevais, MD]; адрес: 117036, Москва, ул. Дмитрия Ульянова, д. 11 [address: 11, Dm. Ulyanova street, Moscow, 117036 Russian Federation]; ORCID: https://orcid.org/0000-0001-5592-4794; eLibrary SPIN: 2459-0540; e-mail: anastassia93@gmail.com

Бельцевич Дмитрий Германович, д.м.н., проф. [Dmitriy G. Beltsevich, MD, PhD, Professor]; ORCID: https://orcid.org/0000-0001-7098-4584; eLibrary SPIN: 4475-6327. e-mail: belts67@gmail.com Абросимов Александр Юрьевич, Д.м.н., профессор [Aleksandr Yu. Abrosimov, MD, PhD]; ORCID: https://orcid.org/0000-0001-8284-9996; eLibrary SPIN: 4089-9502; e-mail: patomorph@endocrincentr.ru Деркач Дмитрий Анатольевич [Dmitriy A. Derkatch, MD]; ORCID: https://orcid.org/0000-0003-2622-8858; eLibrary SPIN: 9549-1557; e-mail: dmitriy_derkatch@mail.ru

Лазарева Анна Александровна, клинический ординатор [Anna A. Lazareva, medical resident]; ORCID: https://orcid.org/0000-0002-1974-7564; eLibrary SPIN: 3940-5767; e-mail: annalazareva15@mail.ru 


\section{ИНФОРМАЦИЯ}

Рукопись получена: 10.06.2021. Одобрена к публикации: 14.07.2021.

\section{ЦИТИРОВАТЬ:}

Шевэ А., Бельцевич Д.Г., Абросимов А.Ю., Деркач Д.А., Лазарева А.А. Клинико-генетическая гетерогенность двусторонней микронодулярной гиперплазии надпочечников // Эндокринная хирургия. — 2021. — Т. 15. — №1. — С. $27-35$. doi: https://doi.org/10.14341/serg12709

\section{TO CITE THIS ARTICLE:}

Chevais A, Beltsevich DG, Abrosimov AYu, Derkatch DA, Lazareva AA. Clinical and genetic heterogeneity of micronodular adrenal hyperplasia. Endocrine surgery. 2021;15(1):27-35. doi: https://doi.org/10.14341/serg12709 